\title{
Nanostructure and EELS Characterization of Catalyst Assisted SiC Nanorods Generated from Single-Walled Carbon Nanotubes
}

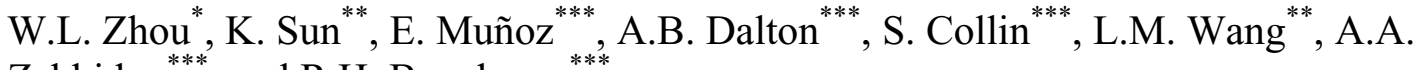 \\ Zakhidov $^{* * *}$, and R.H. Baughman ${ }^{* * *}$
}

*Advanced Materials Research Institute, University of New Orleans, New Orleans, LA 70148

** Dept. of Nuclear Engineering \& Radiological Sciences, University of Michigan, Ann Arbor, MI 48109

${ }^{* * *}$ UTD NanoTech Institute, The University of Texas at Dallas, Richardson, TX 75083

Catalyst assisted nanowires synthesis is widely used as building blocks for assembling nanodevices including FETs, $\mathrm{p}-\mathrm{n}$ diodes, bipolar junction transistors, and complementary inverters, etc [1]. Generally the diameters of catalysts control the size and growth of nanowires. Seeking new catalysts and elucidating the growth mechanisms are crucial to functionalize the nanowires and achieve novel properties. In this paper we present a transmission electron microscopy study of $\mathrm{SiC}$ nanorods fabricated by annealing single-walled carbon nanotubes between Si wafers.

The SiC nanorods were synthesized by annealing sheets of HiPco single-walled nanotubes between $\mathrm{Si}$ wafers at up to $1000^{\circ} \mathrm{C}$ [2]. After cooling down, the $\mathrm{SiC}$ nanorods were scratched on carbon grids for TEM observation. High resolution TEM was performed on the JEOL 2010 equipped with DX Prime EDS microanalysis system. Electron energy loss spectroscopy (EELS) and energy filter TEM (FETEM) were finished using a JEOL 2010F STEM/TEM equipped with Gatan imaging filter (GIF).

Fig. 1a shows a typical $\mathrm{SiC}$ nanorod with a catalyst head. The catalyst controls the nanorod size and growth. The diameters of $\mathrm{SiC}$ nanorods range from several nanometers to several ten nanometers, which are dependent upon the catalyst size. The nanorod is $2 \mathrm{H}-\mathrm{SiC}$ structure with intergrowth of 3C-SiC as shown in HRTEM image in Fig.1b. The catalyst is iron silicide with a tetragonal structure. The $(0001)_{2 \mathrm{H}-\mathrm{SiC}}$ or $(111)_{3 \mathrm{C}-\mathrm{SiC}}$ is nearly parallel to the interface of the catalyst, which is the iron silicide (111) plane as shown in HRTEM image in Fig.1c. There is a thin amorphous layer wrapped on the catalyst surface $(\sim 2 \mathrm{~nm})$ and $\mathrm{SiC}$ nanorod surface $(0.5 \mathrm{~nm})$ as seen in Fig.1a. EELS and FETEM was employed to identify the surface structure. EFTEM images were taken at $4 \mathrm{kX}$ using $2 \times$ binning $(512 \times 512$ pixels $)$ with the second largest objective aperture inserted. Elemental maps of the $\mathrm{C}, \mathrm{Si}$, and $\mathrm{O}$ on the catalyst surface were acquired as shown in Fig.2. It is concluded that a $\mathrm{SiO}_{\mathrm{x}}$ amorphous thin layer formed on the catalyst and nanorod surface. $\mathrm{C}$ amorphous only formed on catalyst surface during the cooling down process. The catalyst assisted $\mathrm{SiC}$ nanorod growth will be further discussed in the presentation.

\section{References}

[1] Y. Huang, X. Duan, Y. Cui. L.J. Lauhon, and C.M. Lieber, Science. 294, (2001) 1313.

[2] E. Muñoz, A.B. Dalton, S. Collins, A.A. Zakhidov, R.H. Baughman, W.L. Zhou, J. He, C.J. O’Connor, B. McCarthy, and W.J. Blau, Chem. Phys. Lett.159 (2002) 397.

[3] The authors gratefully acknowledge the support of a research grant from the Louisiana Board of Regions Contract No. MSF/LEQSF (2001-04)-RII-03. 


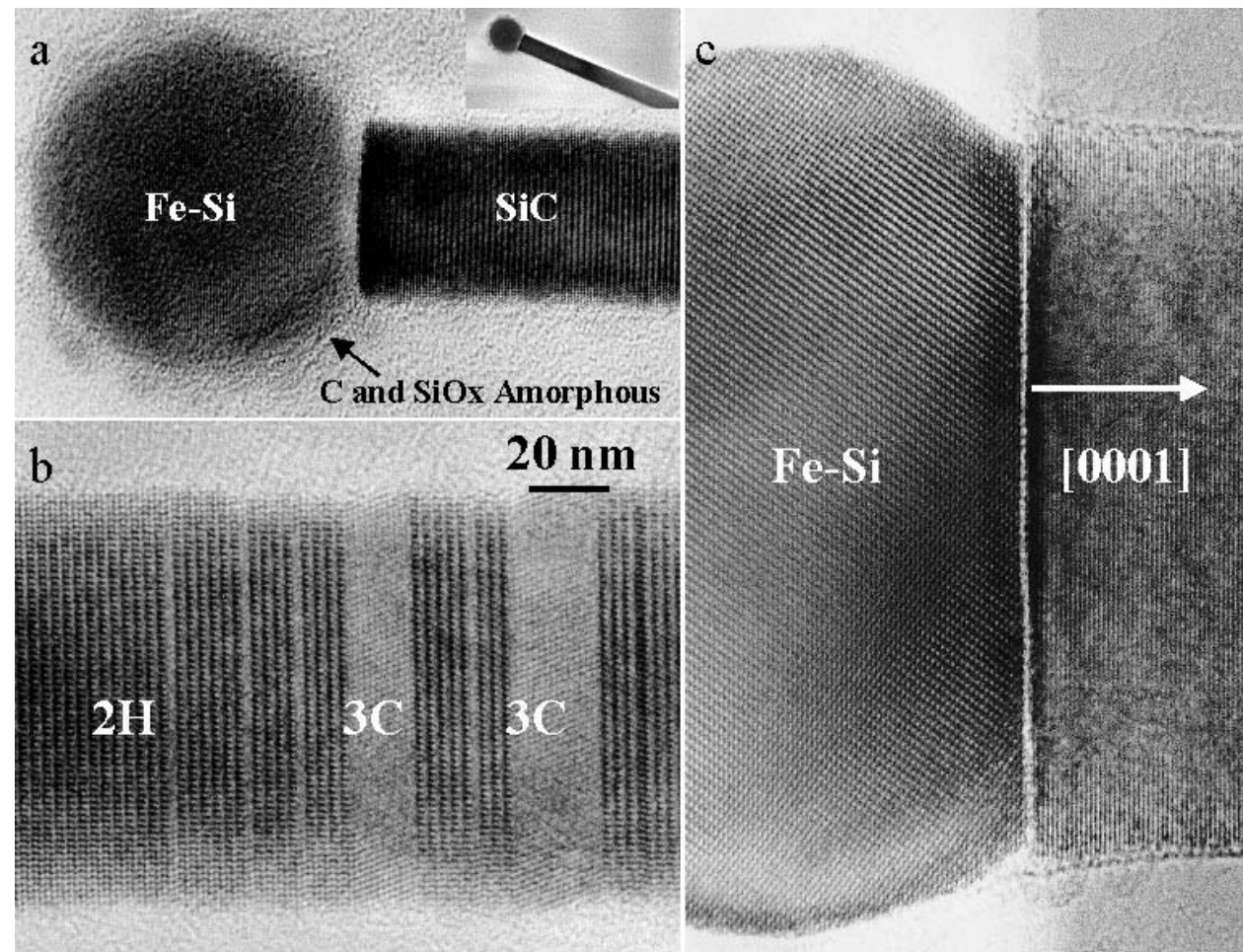

Fig.1 Catalyst assisted $\mathrm{SiC}$ nanorods growth. (a) Iron silicide head controls the $\mathrm{SiC}$ nanorod growth. (b) $\mathrm{SiC}$ nanorod is $2 \mathrm{H}-\mathrm{SiC}$ structure with intergrowth of $3 \mathrm{C}-\mathrm{SiC}$. (c) $(0001)_{2 H-S i C}$ is parallel to (111) of iron silicide.
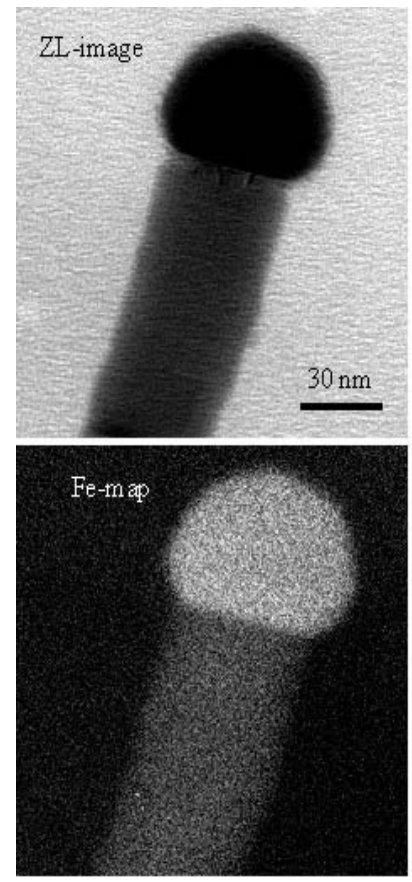
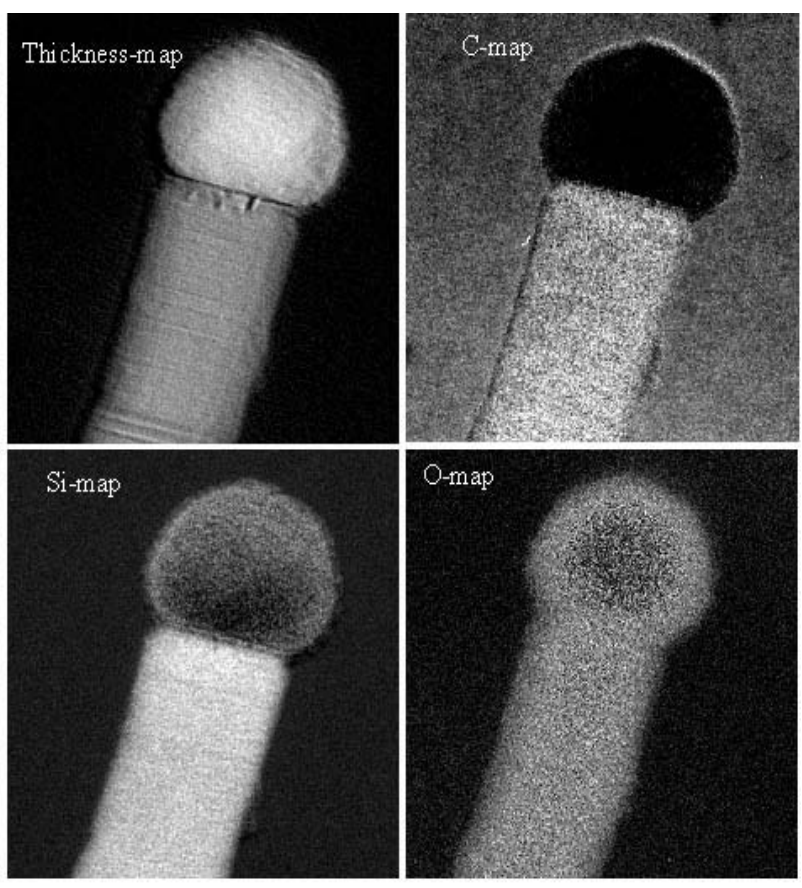

Fig.2 FETEM images of the $\mathrm{C}, \mathrm{Fe}, \mathrm{Si}$, and $\mathrm{O}$. The surface of the catalyst is wrapped with thin $\mathrm{SiO}_{\mathrm{x}}$ and $\mathrm{C}$ amorphous during cooling down process. 